\title{
Focal therapy for prostate cancer-ready to be a standard of care?
}

\author{
Kae Jack Tay ${ }^{1}$
}

Received: 13 April 2021 / Revised: 19 April 2021 / Accepted: 23 April 2021 / Published online: 18 May 2021

(c) The Author(s), under exclusive licence to Springer Nature Limited 2021

With the widespread availability of multiparametric magnetic resonance imaging (mpMRI) and targeted biopsy, we are today able to define the intra-prostatic location and grade of prostate cancer with unprecedented accuracy. It seems natural that the next step would be to precisely target solitary, low volume cancer foci for ablation rather than employ unwieldy whole-gland radical therapies that have much more side effects. Compared to whole-gland therapies, focal therapy has a lesser impact on quality of life $[1,2]$. Is focal therapy thus a new standard of care in treating selected patients for prostate cancer?

Prostate cancer is well-known for its long natural history. Randomized trials in screening and treatment for prostate cancer have shown that a long follow-up with at least 10 or preferably 20 years is necessary to show a meaningful impact on survival [3, 4]. Studies of radical prostatectomy specimens show that most prostate cancers are multifocal and only $15-20 \%$ of localized prostate cancers may be suitable for focal therapy [5]. Despite large strides in the imaging of prostate cancer, the negative predictive value of mpMRI for clinically significant prostate cancer remains between 76 and $89 \%$ depending on one's definition of clinical significance [6]. The majority of focal therapy studies have a short follow-up period that precludes convincing conclusions relating to the rate of metastasis or survival. Among those where mandatory re-biopsies are performed 6-12 months after treatment, the infield recurrence rate of clinically significant cancer is as high as $21 \%$ and that of outfield recurrence is as high as $10 \%$ [7].

The study by Van Son et al. concur with these observations and build upon them further [8]. At a median follow-up of 5 years, the authors report a failure rate of $21 \%$ in 530 men after

Kae Jack Tay

tay.kae.jack@singhealth.com.sg

1 Department of Urology, Singapore General Hospital, SingHealth Duke-NUS Academic Medical Center, Academia, Singapore, Singapore focal therapy, using a composite measure for failure comprising the use of local salvage treatment, metastasis, systemic treatment or progression to watchful waiting. Using a propensity score weighted comparison of men undergoing radiation therapy $(n=440)$, prostatectomy $(n=390)$ or Focal Therapy, Van Son et al found no statistically significant difference in 6-year failure-free survival. Propensity score weighting is a statistical method where weighting is applied to achieve rebalancing in designated variables. In doing so, it seeks to approach the randomized state, where baseline characteristics of the different comparator arms are similar. However, due to the nature of variable selection, it is not possible to address unknown or unmeasured variables during propensity score weighting and the paper should be interpreted with this methodological limitation in mind. Nonetheless, this represents the largest study comparing Focal Therapy to its mainstream counterparts and while we await recruitment and outcomes of randomized trials (e.g., PART and CHRONOS), such prospective registry-based studies provide crucial information with which to counsel patients.

Ultimately, the success of focal therapy depends on patient selection. High quality mpMRI and targeted biopsy is required to grade and localize the cancer accurately. As a highly customized treatment, the indication and outcomes for focal therapy have to be discussed with the patient: is the aim to eradicate all cancer within the prostate or just the clinically significant focus while leaving behind low-grade cancer for active surveillance? The likelihood of being successful may be much higher if the aim were to defer radical treatment or eliminate clinically significant prostate cancer as it appears than if one's aim were to be completely cured. Defining short-medium term success also depends heavily on the follow-up strategy: rather than making posttreatment biopsies mandatory, Van Son et al. performed them for-cause and thus the true rate of cancer persistence after focal therapy might be even higher. Recent expert consensus have concluded that patients should undergo repeat imaging and biopsy at 6-12 months after focal therapy in order ascertain the degree of residual cancer if any [9]. While a mandatory repeat biopsy strategy may 
decrease the short-term failure rate for focal therapy, it may paradoxically increase the long-term success rate from the judicious use of early and appropriate interventions that we know $20-30 \%$ of patients will need.

While the excellent functional outcomes with focal therapy are welcome, patients are unlikely to accept a compromise on good oncological control. Focal therapy should perhaps be viewed as part of a larger strategy: as a possible extender of active surveillance with an aim to defer and select patients with progressive cancer for radical therapy rather than totally avoiding it [10]. Patients with favorable disease characteristics who are highly-involved and compliant in their follow-up are the best candidates for such a strategy and it is perhaps in these patients that focal therapy could eventually be a standard of care.

\section{Compliance with ethical standards}

Conflict of interest The author declares no competing interests.

Publisher's note Springer Nature remains neutral with regard to jurisdictional claims in published maps and institutional affiliations.

\section{References}

1. Tay KJ, Polascik T, Elshafei A, Tsivian E, Jones JS. Propensity score matched comparison of partial to whole gland cryotherapy for intermediate-risk prostate cancer: an analysis of the cold registry data. J Urol. 2016;195:e199.
2. Scheltema MJ, Chang JI, Bohm M, van den Bos W, Blazevski A, Gielchinsky I, et al. Pair-matched patient-reported quality of life and early oncological control following focal irreversible electroporation versus robot-assisted radical prostatectomy. World J Urol. 2018;36:1383-9.

3. Hugosson J, Roobol MJ, Månsson M, Tammela TLJ, Zappa M, Nelen V, et al. A 16-yr follow-up of the European randomized study of screening for prostate cancer. Eur Urol. 2019;76:43-51.

4. Wilt TJ, Vo TN, Langsetmo L, Dahm P, Wheeler T, Aronson WJ, et al. Radical prostatectomy or observation for clinically localized prostate cancer: extended follow-up of the prostate cancer intervention versus observation trial (PIVOT). Eur Urol. 2020;77:713-24.

5. von Hardenberg J, Borkowetz A, Siegel F, Kornienko K, Westhoff N, Jordan TB, et al. Potential candidates for focal therapy in prostate cancer in the era of magnetic resonance imaging-targeted biopsy: a large multicenter cohort study. European Urology Focus. 2020;S2405-4569:30274-1.

6. Ahmed HU, El-Shater Bosaily A, Brown LC, Gabe R, Kaplan R, Parmar MK, et al. Diagnostic accuracy of multi-parametric MRI and TRUS biopsy in prostate cancer (PROMIS): a paired validating confirmatory study. Lancet 2017;389:815-22.

7. Tay KJ. Prostate focal therapy: the rule or exception? Curr Opin Urol. 2018;28:512-21.

8. van Son MJ, Peters M, Reddy D, Shah TT, Hosking-Jervis F, Robinson S, et al. Conventional radical versus focal treatment for localized prostate cancer: a propensity score weighted comparison of 6-year tumour control. Prostate Cancer Prostatic Dis. 2021.

9. Tay KJ, Amin MB, Ghai S, Jimenez RE, Kench JG, Klotz L, et al. Surveillance after prostate focal therapy. World $J$ Urol. 2019;37:397-407.

10. Tay KJ, Scheltema MJ, Ahmed HU, Barret E, Coleman JA, Dominguez-Escrig J, et al. Patient selection for prostate focal therapy in the era of active surveillance: an International Delphi Consensus Project. Prostate Cancer Prostatic Dis. 2017;20:294-9. 